and/or surgical treatment depends on the Stage of the CE-infection, not on the general condition of the patient.

\section{ANTIBIOTICS STUDY ON STAPHYLOCOCCUS SPP. STRAINS ISOLATED FROM VENOUS AND URINARY CATHETERS IN NICU OF HAMADAN HOSPITALS, WEST RAN}

doi:10.1136/archdischild-2012-302724.0888

'R Yousefimashouf, ${ }^{2 N}$ Molazadeh. 'Department of Microbiology, Research Center for Molecular Medicine of Hamadan University of Medical Sciences; ${ }^{2}$ Faculty of Medicine, Hamadan University of Medical Sciences, Hamadan, Iran

Background and Aim Staphylococcus coagulase negative strains are colonized on epiderm and distribute in environment and outer bodies apparatus such as protez and intera-venous catheters. The aim of this study was the frequency of Staphylococcus coagulase negative isolated from venous and catheters children hospitalized in NICU of Hamadan hospitals and determination of antibiotics resistance patterns in Hamadan, the west of Iran

Methods We collected 108 samples randomly from patients who were hospitalized in NICU hospitals of Hamadan and they needed to venous or urinary catheters. One specimen of each patient was taken and inoculated into carrier transported media and transferred to bacteriology laboratory to identification of strains. Antibiogram was performed by Kirby-Bauer method. Data was analyzed using SPSS 15 software.

Results Out of 108 tested samples, $32.7 \%$ of patients had urinary catheter and $67.3 \%$ had venous catheter. $28 \%$ of tested samples had positive culture. The positive cases were significantly found in those children who had been used catheter more than 48 hours $(P=0.00)$. From the positive cases, Staphylococcus epidemidis (40.4\%), Acinetobacter baumannii (10.6\%) and E. coli (8.5\%) were the most common isolates. The most rate of resistance of Staphylococcus epidemidis was against to erythromycin and ampicillin. The most rate of sensitivty of E. coli was against to gentamicin and Psuedomonas aeruginosa to ciprofloxacin.

Conclusion Our results showed the high contamination in used catheters particularly in those patients who needed to catheter for long time. We also indicated the high drug resistance in strains isolated from catheters.

\section{ETIOLOGIES OF THE STATUS EPILEPTICUS IN CHILDREN HOSPITALISED IN THE PICU OF THE UNIVERSITY HOSPITAL CENTER OF ORAN (ALGERIA)}

doi:10.1136/archdischild-2012-302724.0889

D Boumendil, MA Negadi, K El Halimi, H Bouguetof, ZC Mentouri. Pediatric Intensive Care Unit, Faculty of Medicine - Oran University, Oran, Algeria

Background and Aims Status Epilepticus is one of the most frequent neurological emergencies in Pediatrics, that can involve the vital and functional prognosis in the short and long term.

The Aim of this study is to determine epidemiological, causative, diagnostic aspects and to evaluate the therapeutic means.

Methods In this prospective study we analyse 214 children with status epilepticus between January 2008 and December 2010.

Results The mean age is 04 years (min: 28 days - max: 15 years) with a sex ratio equal to 1.3 . $60 \%$ of cases was febrile. $81 \%$ of the seizures are generalized. The differents etiologies are: Epilepsy: 59 (27.5\%); Occasional seizures: 69 (32.24\%); Infections of central nervous system (CNS): 53 (24.7\%) (26 méningitidis and 27 méningoencéphalitis); Febrile seizures: 24 (11\%); Indeterminate cause: 09 (04\%).

It was noted a long delay between the onset of clinical manifestations and world-renowned workplace support. The drugs used are represented by injectable diazepam and phenobarbital. In terms of support, $46 \%$ need artificial ventilation. In this series the mortality is $22 \%$.

Conclusion The vital and functional prognosis could be improved by better prevention and effective treatment of infectious diseases, a reduction of the period of support and better ways of resuscitation

\section{URINARY TRACT INFECTION AND PREDISPOSING FACTORS IN CHILDREN}

doi:10.1136/archdischild-2012-302724.0890

'M Naseri, ${ }^{2}$ SA Alamdaran. ${ }^{1}$ Pediatric Nephrology; ${ }^{2}$ Radiology, Mashhad University of Medical Sciences, Mashhad, Iran

Objective This study was designed to determine the predisposing factors in children with symptomatic urinary tract infection (UTI) according to age and gender.

Material and Methods We reviewed prospectively 183 pediatric patients with symptomatic UTI admitted to emergency department or referred to nephrology clinic from November 2002 through July 2005. All patients underwent renal ultrasonography and voiding-cystouretherography or radionuclide cystography. Diuretic renal scan or intravenous pyelography (IVP) was performed in those with urinary system dilatation. Urodynamic studies were done in patients with normal radiologic findings and recurrent infections or urinary-intestinal symptoms.

Findings Of 183 patients, 130 cases (71\%) were female and 53 patients (29\%) male. Most of the patients (61.9\%) were between 2-24 months old ( $\mathrm{P}=0 / 001)$. Vesicoureteral reflux (VUR) was the most common predisposing factor in both genders $(46.9 \%$ in girls and $48.9 \%$ in boys). Voiding dysfunction in girls and urinary obstruction in boys were found with a significant difference $(\mathrm{P}=0 / 03$ for both). In all age groups, except patients $\leq 1$ month, the most common predisposing factor was reflux. Reflux, urinary obstruction and nephrolithiasis were found with a significant difference in 2-24 months age group ( $\mathrm{P}=0 / 001$ for all).

Conclusion In our study vesicoureteral reflux (VUR) was as common in boys as in girls, and suggested urolithiasis as a significant UTI predisposing factor. This study showed that voiding dysfunction in girls and urinary obstruction in boys are as significant predisposing factors. We suggest urodynamic studies in patients with normal radiologic findings and recurrent infections or urinaryintestinal symptoms

\section{ACUTE PYELONEPHRITIS AND DIAGNOSTIC PARAMETERS}

doi:10.1136/archdischild-2012-302724.0891

'VN Stavileci, 'M Begolli, ${ }^{2} \mathrm{~A}$ Keka. ${ }^{1}$ Nephrology, University Clinical Centre of Kosova, Pediatric Clinic; ${ }^{2}$ University Clinical Centre of Kosova, Prishtina, Kosovo

Introduction Diagnosis and the right time management of Pyelonephritis are extremely important especially if we consider the risk of permanent kidney damage.

Objectives and research To analyze the clinical and laboratory signs and radiological presentation of the disease in children diagnosed with acute pyelonephritits during year 2010 in Pediatric clinic, Nephrology Unit.

Methods Among cases admitted to Nephrology Unit diagnosed as pyelonephritis acuta, during 2010, we analyzed presentation symptoms by age, inflammatory laboratory results, protein degradation products, urine and kidney ultrasound findings.

Results Among of 83 cases with urinary tract infections, 32.5\% were diagnosed as pyelonephritis acuta. More frequent among male infants and preschool age and on female school age. $29.6 \%$ of the cases were male and $70.3 \%$ of the cases were female. Inflammatory parameters were high in $88.8 \%$ of cases and the value of above SE: $100 \mathrm{~mm} / \mathrm{h}$ was in $20.8 \%$ of cases. Dominated presentation 